\title{
Method for energy-efficient planning of the industrial processes
}

\author{
Gabriel Frumuşanu ${ }^{1, *}$, Nicolae Badea ${ }^{2}$, Cezarina Afteni ${ }^{1}$, and Alexandru Epureanu ${ }^{1}$ \\ ${ }^{1}$ Dunărea de Jos University of Galaţi, Department of Manufacturing Engineering, Domnească Street \\ 111, 800201 - Galaţi, Romania \\ 2 Dunărea de Jos University of Galați, Deppartment of Automatic Control \& Electrical Engineering, \\ Ştiinţei Street 2, 800210 - Galaţi, Romania
}

\begin{abstract}
In industry, process planning has a major impact on manufacturing performance. This paper proposes a method aiming to increase the efficiency in process planning, more specific in selecting the set of plans with high potential of performance. The method works on the base of processes classification after their energy efficiency. It is applied for processes grouped in the same utility area, and involves the definition of energy classes. A case study, having as purpose to prove the feasibility and effectiveness of the proposed method is also presented. Energy labelling may further become an efficient tool enabling the manufacturing process optimization from energetic point of view.
\end{abstract}

\section{Introduction}

The numerous dramatic events from recent years (floods, droughts, devastating storms), due to environment changes issuing from the negative impact of human activities, have brought in front of human kind the new challenge synthesised in the concept of sustainable development, one of its key-features being energy efficiency. The industrial activity, deployed through industrial processes, involves by its nature the use of a consistent share from the energy consumed at global level. Hereby, the energy efficiency of the industrial process is a top priority subject.

The energy needed to perform an industrial process has two main components: the energy required for underlying the process and the energy consumed inside the process, for producing its utile result (e.g. machining a surface, welding two parts etc.). Although the machine and the industrial process have no reason of existing one without another, the existence of two energy components leads to the possibility of speaking about energy efficiency at machine level and process level, respectively. Furthermore, separately rating the energy efficiency of machine and industrial process makes sense, because the same process may be performed on different machines, as well as the same product may be obtained using different processes. In the first case, one might need to choose a machine for running a process (having the energy efficiency as criterion), while in the second, the process for obtaining a product is the choice to be made.

\footnotetext{
*Corresponding author: gabriel.frumusanu@ugal.ro
} 
The researches presented in literature dedicated to energy efficiency in manufacturing approach the problem at two distinct levels. First one is the level of the natural process, which may be included in different industrial processes. For example, [1] starts from the energetic components of material removal in the cutting process and consider the consumed energy as a function of five parameters (cutting speed, feed rate, tool nose radius, cutting edge radius and rake angle). A similar approach in energetic modelling the cutting process is presented in $[2,3]$. The second level is the one of the machine - process couple. In this case, the energy components consumed by both machine and process are put together and the efficiency is globally assessed when milling [4], turning \& grinding [5], grinding [6]. More comprehensive approaches are detailed in [7,8].

In this paper, the energy efficiency problem is approached at an intermediary level, addressing the ensemble formed by the natural process and consumables needed for running the process. By consumables we mean, for example, the cutting tool and the cooling liquid, in the case of a cutting process, the forming die and the lubricant (forming process), the electrode and the inert gas for shield (arc welding), etc. Paper target is to develop a method for energy-efficient planning of industrial processes. Without generality restraining, for sake of discourse clarity we will further address to manufacturing processes (one of the most relevant category of industrial processes). Because any product can be manufactured by following diverse process plans, in practice one have to find the optimal solution, by choosing between the plans with highest performance, which should apply processes with optimality potential. As energy efficiency is addressed here for performance assessment, the tool we propose in order to find the appropriate processes consist in grouping them in energy efficiency classes, defined for procedures utility areas. Next section presents the method proposed for energy-efficient planning, while the third presents a case-study for energy labelling the industrial process. Last section is for conclusion.

\section{Proposed method for processes planning}

\subsection{Planning stages}

In manufacturing companies, the first action to be followed after receiving an order for a certain product is processes planning, which has a major impact on manufacturing performance. Processes planning is supposed to cover three successive stages, namely selection, programming, and analysis.

Selection starts by decomposing the order in jobs (at first level) and, subsequently, each job in tasks (at second level), see Figure 1. Job means manufacturing a product component, while task means an elementary manufacturing process (e.g. manufacturing a hole, a key slot or a thread). After order decomposition, procedures available in the considered workshop are assigned to the tasks needing to be achieved. Selection purpose is to generate set of sub-optimal process plans. Here, by industrial process we mean the actual deployment of a natural processes set, artificially provoked and taking part correlated, conducted towards an expected result (generically meaning task accomplishment). At process input there are four items: materials, energy, time and information. Any process deploys on a logistic bed, here designated as workstation. The methodological algorithm according to which the process takes part is called procedure.

Programming consists in establishing the actual conditions for running the selected plans, namely values for all parameters of the involved procedures, as well as the setup of workstations assigned in this purpose.

Analysis aims to choose the optimal plan, after the considered performance criterion. This can be done once and for all, before manufacturing beginning, or along the order 
accomplishment, depending on actual circumstances. Second option takes into account possible modifications of manufacturing conditions, which may lead to the situation when another sub-optimal plan becomes optimal, instead of the previously chosen one.

The proposed method distinguishes by the particular manner for performing the first planning stage, namely selection.

\subsection{Selection}

As jobs decomposition in tasks is not unique and procedures assignment to tasks either, it follows that more variants of processes planning can be generated. A process plan variant result after selecting from a task pool the tasks required for jobs achievement, the assigned manufacturing procedures from a procedures pool and the support workstations from a workstations pool, and after combining tasks and procedures in processes (Figure 1). As it can be noticed, a procedure may be used in more processes and a workstation may support different procedures.

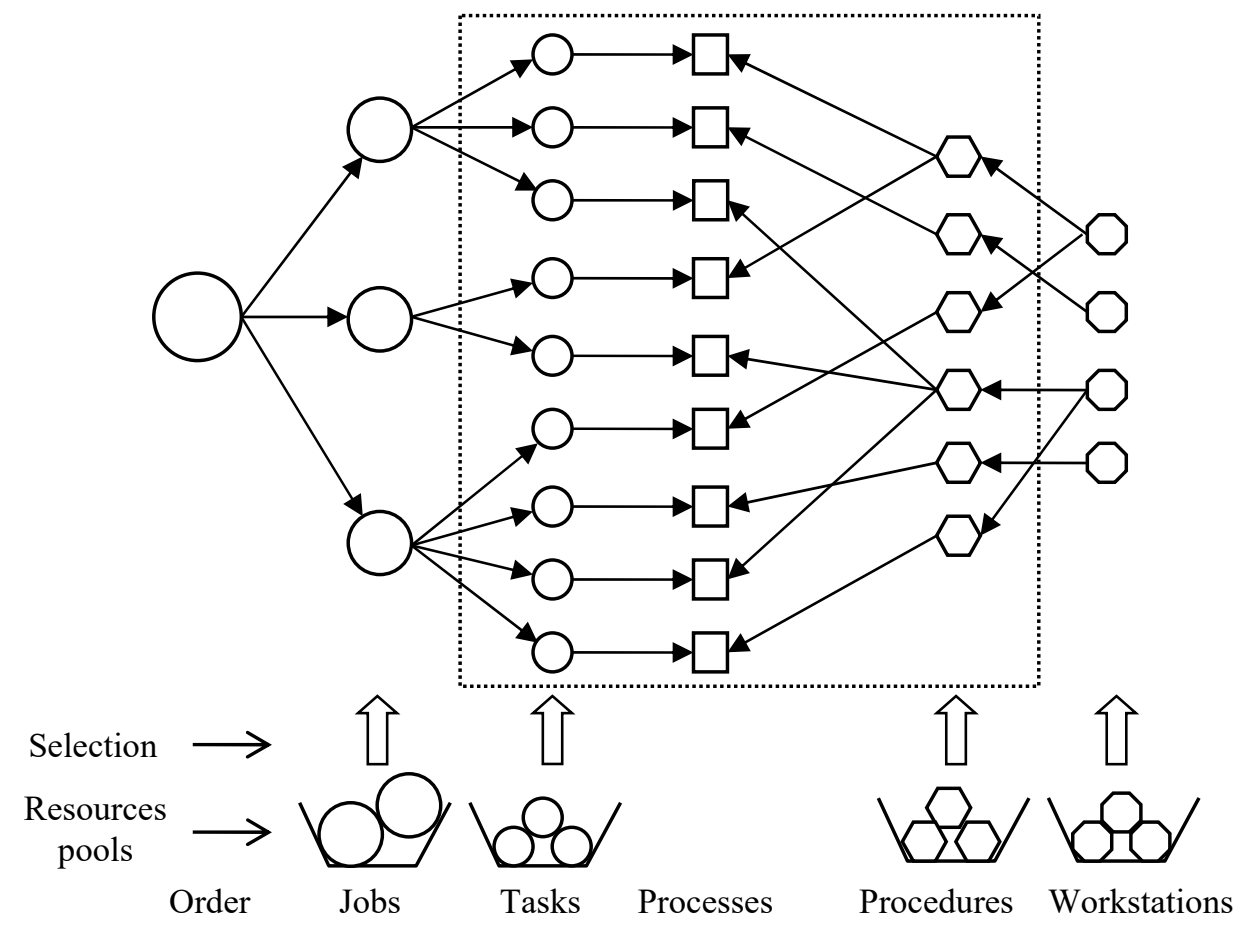

Fig. 1. Graf illustrating a generic variant of process planning.

The final action from selection stage is the assessment of all available plan variants, followed by choosing the set of sub-optimal plans, having high potential of performance. For finding the sub-optimal plans, an appropriate assessment tool is necessary. In our vision, such tool is a system of performance classes. In this paper we will refer, in particular, to energy efficiency classes.

The assessment is proposed to be realized based on energy labelling, which is a classification (which highlights the similitude between processes) and not by their measurement (which highlights the difference between them), as currently practiced. In our vision, classification is a more appropriated tool than measurement, because it works faster and is more adequate when addressing to process plans potential. 


\subsection{Processes classification}

The proposed classification supposes efficiency assessment for the processes from the same utility area, based on processes similitude and reflecting the potential performance they can more likely reach. Processes classification consists in identifying the energy class to which a given process belongs.

\subsubsection{Utility area definition}

In order to build an energy classes system, a precursory action needs to be accomplished. It consists in defining utility area, materializing the connection between procedure and task the last one being addressed in general sense. For example, task might mean manufacturing a certain type of surface (plane exterior, cylindrical interior, thread etc.), manufacturing with a given precision, or manufacturing surfaces with specified roughness.

One should notice that the same task can be achieved by different procedures (e.g. a cylindrical interior surface can be machined by drilling, boring, turning, grinding), while the same procedure may cover different tasks (milling can be used for generating plane surfaces, profiled surfaces or threads). Hereby, the utility area may be formed by $i$ ) the tasks which can be achieved by applying a given procedure (e.g. the tasks $t_{1}, t_{2}$ and $t_{4}$ for the procedure $P_{l}$, Figure 2), or ii) the procedures covering a certain task (e.g. the procedures $P_{2}, P_{3}$ and $P_{4}$ for the task $t_{3}$, Figure 2). First of the two utility area definitions has application in product design, when adjusting the tasks to procedure potential. The second is necessary in product manufacturing, if choosing among the procedures covering a task; as this paper is about process planning, from now-on this will be the considered definition of utility area.

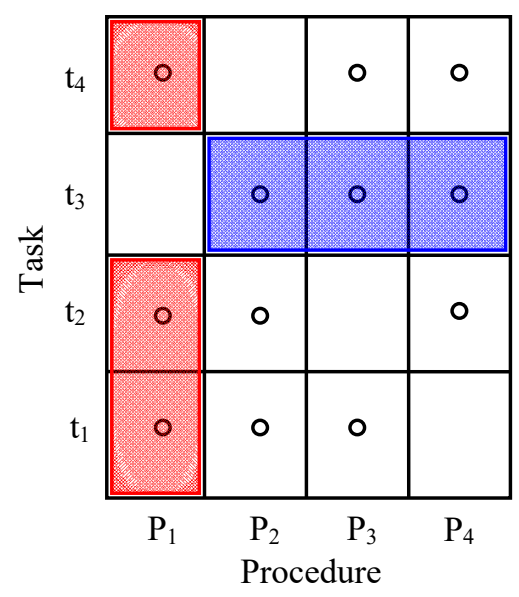

Fig. 2. Utility areas definition.

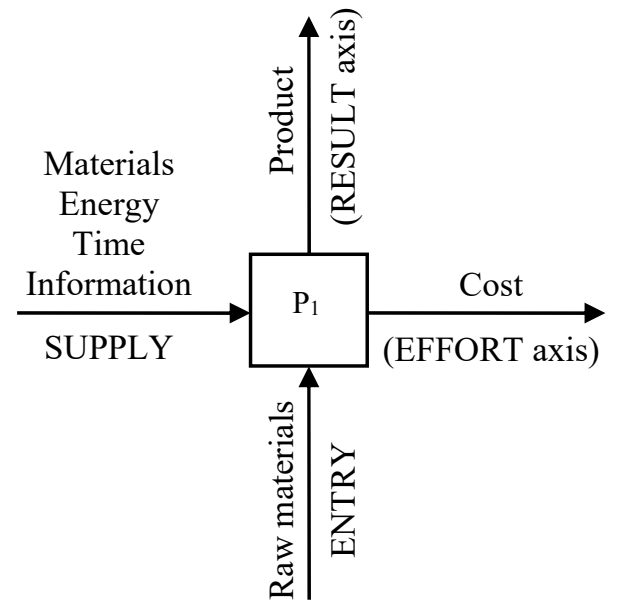

Fig. 3. Procedure flowcharts.

\subsubsection{Energy classes definition}

If looking now to a procedure (for sample $P_{l}$ ), then we can assign it two axis, namely Effort axis and Result axis (see Figure 3). Along the first one, as input there is the supply (consisting in materials, energy, time and information) and as output - the corresponding cost. As second axis entry there are raw materials (the workpiece but also materials with 
technologic role), which are necessary to obtain the product by applying the processing algorithm.

Manufacturing processes classification after their energy efficiency is approached based on the general efficiency definition:

$$
\text { Efficiency }=\frac{\text { Result }}{\text { Effort }}
$$

The manner of defining and representing the energy classes for the procedures covering a task (for sample $t_{3}$ ) is presented in Figure 4.

In the depicted diagram, each generic process $P$ is represented as a point. The effort involved to run the process and the result issued from this effort, both measured through appropriate indicators, are giving $P$ coordinates. Because procedure and task parameters may vary, $P$ position may also change. The locus of points corresponding to all possible values of parameters for a given procedure defines a close domain assigned to it in the graphic representation. By introducing a third coordinates axis, circular, the energy efficiency of a process is reflected through the angle made by $O P$ segment with Effort axis, as suggested on the picture. The energy efficiency classes can be defined now by dividing in several sectors the angular domain comprised between the lines corresponding to extreme efficiencies (inferior/superior), as they can be revealed at classification moment.

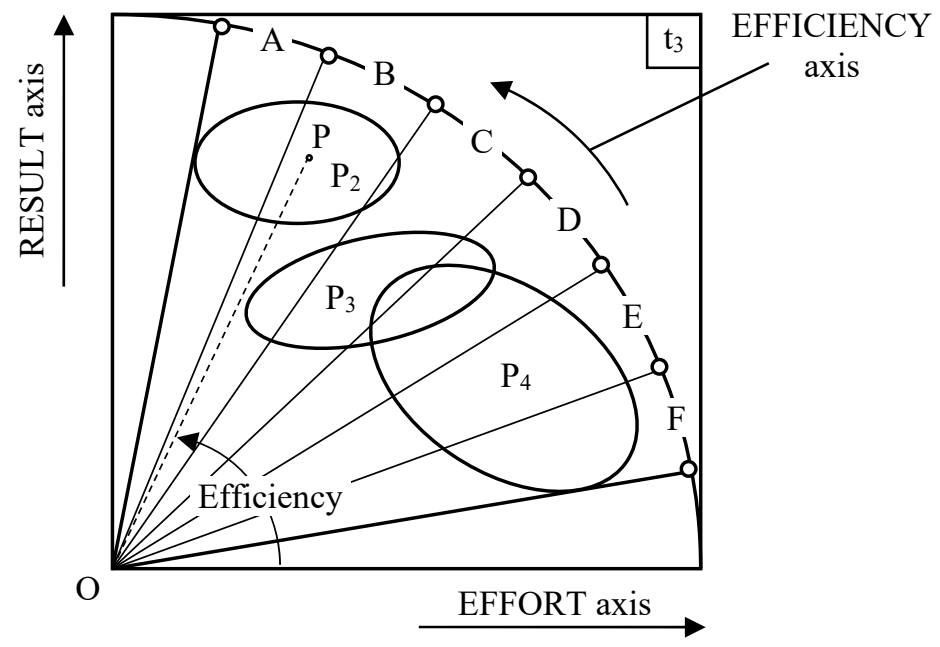

Fig. 4. Effort - Result - Efficiency diagram for $t_{3}$ task.

By similitude with products energy-labelling, according to current EU directive [9], the efficiency classes for manufacturing processes are labelled by letters from $A$ (the most efficient) to $F$ (the least efficient, if considering six classes). The label of a certain procedure is given by classes which its domain covers. Some remarks have to be made:

- The assigned efficiency class is not necessarily unique; actually it may vary depending on procedure parameters values. For example, the efficiency class of $P_{3}$ process (Figure 4) is $B-D$.

- The same process might belong to several utility areas, having different efficiency labels in each case. Moreover, in the same efficiency class one may find more procedures, from different tasks.

- Despite the efficiency labelling is a local classification, it has the same structure, no matter of the considered task or procedure. 
- The possible enhancement of procedures efficiency, subsequent to the moment when the efficiency classes were defined, may lead to occurrence of new classes, above $A$, which will be denoted by $A+, A++$ etc., until the entire classification will be updated.

\section{Case study}

As case study, we present the application of the algorithm for developing the energy classes system for the utility area resulted by associating the task "Cut-off from bar" with five procedures supposed to be available for achieving it, as specified in Figure 5.

Here by cut-off we mean to detach a material piece from remaining bar by removing the layer of material previously existing between two plane parallel transversal sections of the bar. For simplicity, we accepted to characterize the task and each of the considered procedures by two features, as mentioned in Figure 5. For sample, in task case, chosen features are material and dimensions (more specific, hardness and transversal dimension, respectively).

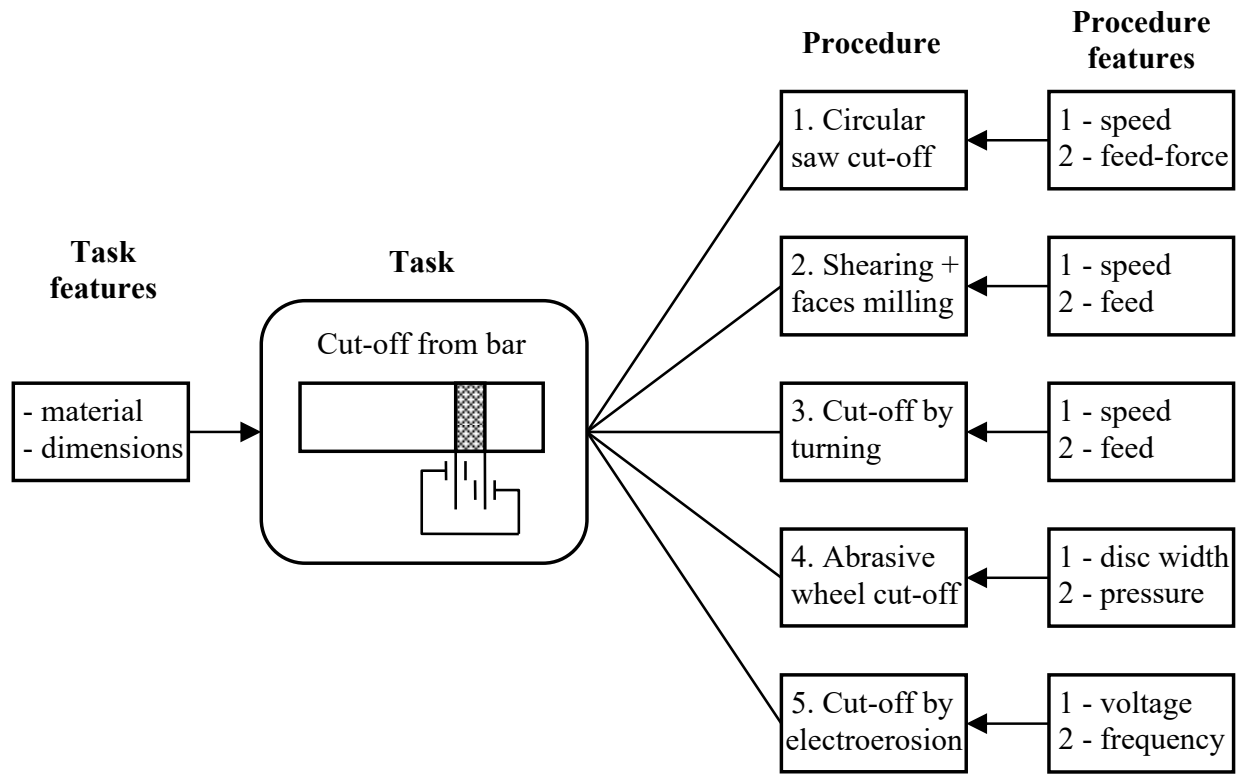

Fig. 5. Utility area for iron bar cut-off.

If speaking in general, after selecting the available procedures for achieving the considered task, the table of the characteristic processes need to be built. Each characteristic process results by combining the task (with actual features) with one of the procedures (also with actual features). The number of representative values for task and procedures features depends on the extension of their variation domains. The table's number of rows is equal to characteristic processes' number.

The process energy efficiency $E E$ is defined as:

$$
E E=\frac{S A}{E C},
$$

where $S A$ means the area of the frontal surface delimiting the detached piece of material, expressed in $\mathrm{cm}^{2}$, while $E C$ is the energy consumed in cut-off process, in Wh, resulting in general, as sum of four components: 


$$
E C=E C_{p}+E C_{p m}+E C_{a m}+E C_{t} .
$$

In relation (3) $E C_{p}$ means the energy directly consumed in the process while $E C_{p m}, E C_{a m}$ and $E C_{t}$ are the amounts of energy embedded in processed material, auxiliary materials and tool share consumed in the process, respectively.

Excerpts from the representative processes table, generated as artificial dataset for the addressed case study, are presented in Table 1.

Table 1. Energy efficiency of representative processes.

\begin{tabular}{|c|c|c|c|c|c|c|c|}
\hline \multirow{2}{*}{$\begin{array}{l}\text { Crt. } \\
\text { no. }\end{array}$} & \multicolumn{2}{|c|}{ Task features } & \multirow{2}{*}{$\begin{array}{c}\text { Procedure } \\
\text { number }\end{array}$} & \multicolumn{2}{|c|}{ Procedure feature value } & \multirow{2}{*}{$\begin{array}{c}\text { Sectioned } \\
\text { area } \\
{\left[\mathrm{cm}^{2}\right]}\end{array}$} & \multirow{2}{*}{$\begin{array}{c}\text { Energy } \\
\text { consumed } \\
{[\mathrm{Wh}]}\end{array}$} \\
\hline & $\begin{array}{c}\text { material / HB } \\
{[\mathrm{MPa}]}\end{array}$ & $\begin{array}{c}\text { dimension } \\
{[\mathrm{mm}]}\end{array}$ & & Feature 1 & Feature 2 & & \\
\hline 1 & steel / 1700 & 40 & 1 & $1400 \mathrm{~m} / \mathrm{min}$ & $1000 \mathrm{~N}$ & 12.56 & 2.56 \\
\hline 2 & brass / 650 & 60 & 2 & $150 \mathrm{~m} / \mathrm{min}$ & $1.2 \mathrm{~mm} / \mathrm{rot}$ & 28.27 & 1.4 \\
\hline 3 & steel / 2000 & 50 & 3 & $70 \mathrm{~m} / \mathrm{min}$ & $0.2 \mathrm{~mm} / \mathrm{rot}$ & 19.63 & 8.17 \\
\hline 4 & steel / 1700 & 55 & 4 & $1.6 \mathrm{~mm}$ & $115 \mathrm{MPa}$ & 23.76 & 3.2 \\
\hline 5 & steel / 2300 & 25 & 5 & $40 \mathrm{~V}$ & $4600 \mathrm{~Hz}$ & 4.91 & 4.1 \\
\hline 6 & bronze / 900 & 50 & 1 & $1400 \mathrm{~m} / \mathrm{min}$ & $800 \mathrm{~N}$ & 19.63 & 4.36 \\
\hline 7 & steel / 2000 & 60 & 2 & $145 \mathrm{~m} / \mathrm{min}$ & $1 \mathrm{~mm} / \mathrm{rot}$ & 28.27 & 8.83 \\
\hline 8 & brass / 650 & 70 & 3 & $120 \mathrm{~m} / \mathrm{min}$ & $0.25 \mathrm{~mm} / \mathrm{rot}$ & 38.48 & 9.24 \\
\hline 9 & steel / 2300 & 50 & 4 & $2.5 \mathrm{~mm}$ & $130 \mathrm{MPa}$ & 19.63 & 6.54 \\
\hline 10 & steel / 2500 & 35 & 5 & $40 \mathrm{~V}$ & $4600 \mathrm{~Hz}$ & 9.62 & 6.87 \\
\hline
\end{tabular}

By graphical representing the characteristic processes in $(E C, S A)$ coordinate we obtain the general diagram from Figure 4, particularised in the case of cut-off utility area (Figure 6). If splitting in six the angular domain comprised between the points giving the most efficient process and the less efficient one, then the efficiency domain for classes $\mathrm{A} \ldots \mathrm{F}$ results (see Table 2). After inscribing the points corresponding to the same procedure in a closed curve we can further assess the energy class(es) for each procedure (see Table 3 ).

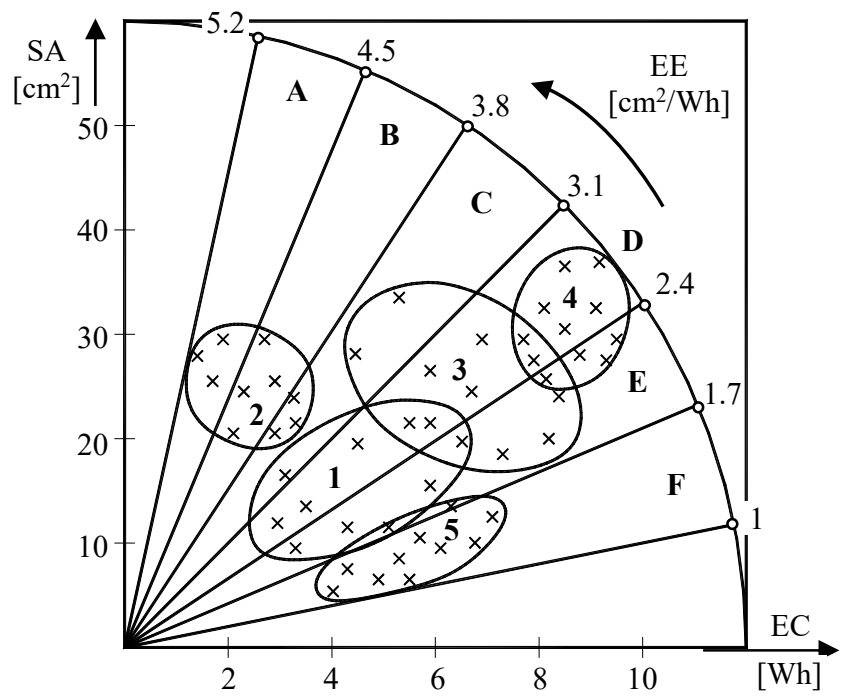

Fig. 6. Energy classes for cut-off process.

It should be noticed that, due to harmonisation between general interest (sustainability) and particular interest (economic efficiency) through legal regulations (e.g. EU directives), the use of manufacturing procedures corresponding to lower energy efficiency classes might become prohibitive. 
Table 2. Energy classes domains.

\begin{tabular}{|c|c|}
\hline Energy class & $\begin{array}{c}E E \text { domain } \\
{\left[\mathrm{cm}^{2} / \mathrm{Wh}\right]}\end{array}$ \\
\hline $\mathrm{A}$ & $4.5 \ldots 5.2$ \\
\hline $\mathrm{B}$ & $3.8 \ldots 4.5$ \\
\hline $\mathrm{C}$ & $3.1 \ldots 3.8$ \\
\hline $\mathrm{D}$ & $2.4 \ldots 3.1$ \\
\hline $\mathrm{E}$ & $1.7 \ldots 2.4$ \\
\hline $\mathrm{F}$ & $1.0 \ldots 1.7$ \\
\hline
\end{tabular}

Table 3. Procedures energy classes.

\begin{tabular}{|l|c|}
\hline \multicolumn{1}{|c|}{ Procedure } & $\begin{array}{c}\text { Energy } \\
\text { class }\end{array}$ \\
\hline 1. Circular saw cut-off & $\mathrm{C}-\mathrm{E}$ \\
\hline 2. Shearing + faces milling & $\mathrm{A}-\mathrm{C}$ \\
\hline 3. Cut-off by turning & $\mathrm{C}-\mathrm{E}$ \\
\hline 4. Abrasive wheel cut-off & $\mathrm{D}-\mathrm{E}$ \\
\hline 5. Cut-off by electroerosion & $\mathrm{E}-\mathrm{F}$ \\
\hline
\end{tabular}

\section{Conclusion}

This paper proposes a method aiming to increase the efficiency in process planning. The method works on the base of processes classification after their energy efficiency. It is applied for processes grouped in the same utility area, and involves the definition of energy classes with the help of a newly imagined graphical representation: the Effort - Result Efficiency diagram.

The performed case study shows that:

- The method has a wide potential of applicability.

- Both method implementation and building of energy classes system afferent to a utility domain are feasible.

- The method is effective, the differences between procedures being significant from energy efficiency point of view.

- Method application may lead to an enhancement of processes performance by facilitating process planning optimization.

The authors acknowledge the use of the research facilities developed by RO-054 Project, financed through EEA Grant 2009 program, in performing the research presented in this paper.

\section{References}

1. H. S. Park, T. T. Nguyen, J. C. Kim, Transactions of FAMENA, XL-1, 1 (2016).

2. E. Abele, S. Braun, P. Schraml, Procedia CIRP 29, 251 (2015).

3. J. Ma, X. Ge, S.I. Chang, S. Lei, Int. J. Adv. Manuf. Technol., 74, 1701 (2014).

4. H. S. Yoon, J. Y. Lee, M. S. Kim, S. H. Ahn, Journal of Cleaner Production, 78, 54 (2014).

5. Y. Guo, J. R. Duflou, B. Lauwers, Int. J. Adv. Manuf. Technol., 75, 503 (2014).

6. P. C. Priarone, Int J Adv Manuf Technol, 86, 2107 (2016).

7. K. Salonitis, P. Ball, Procedia CIRP - Manuf. Systems, 7, 634 (2013).

8. L. Zhou, J. Li, F. Li, Q. Meng, J. Li, X. Xu, Journal of Cleaner Production, 112, 3721 (2016).

9. Directive 2010/30/EU on the indication by labelling and standard product information of the consumption of energy and other resources by energy-related products. 